\title{
Erratum to: Combined effect of hydrogen sulphide donor and losartan in experimental diabetic nephropathy in rats
}

\author{
Manpreet Kaur, Shilpi Sachdeva, Onkar Bedi, Tavleen Kaur and Puneet Kumar*
}

The original version of this article [1] unfortunately contained mistakes to the figure legends and was missing Figure 7 (Fig. 1 here). The corrected legends are as follows:

Fig. 1 Experimental Protocol design.

Fig. 2 Effect of NaHS on blood glucose level in STZ treated rats. ${ }^{\mathrm{a}} P<0.05$ versus vehicle treated, ${ }^{\mathrm{b}} P<0.05$ versus $\left[\right.$ STZ (45)] treated group, ${ }^{\mathrm{c}} P<0.05$ versus [STZ (45) + NaHS (10)] treated group, ${ }^{\mathrm{d}} P<0.05$ versus [STZ (45) + NaHS (30)] treated group. STZ = Streptozotocin, $\mathrm{NaHS}=$ Sodium hydrosulphide, LOS = Losartan, DL-p = DL-propargylglycine.

Fig. 3 Effect of NaHS and losartan on Lipid Peroxidation in STZ treated rats. ${ }^{a} P<0.05$ versus vehicle treated, ${ }^{\mathrm{b}} P<0.05$ versus [STZ (45)] treated group, ${ }^{\mathrm{c}} P<0.05$ versus [STZ (45) + NaHS (10)] treated group, ${ }^{\mathrm{d}} P<0.05$ versus [STZ (45) + NaHS (30)] treated group, ${ }^{\mathrm{e}} P<0.05$ versus $[\mathrm{STZ}(45)+\mathrm{LOS}(5)]$ treated group. STZ = Streptozotocin, NaHS = Sodium hydrosulphide, LOS = Losartan, DL-p = DL-propargylglycine.

Fig. 4 Effect of NaHS and losartan on Reduced Glutathione in STZ treated rats. ${ }^{\mathrm{a}} P<0.05$ versus vehicle treated, ${ }^{\mathrm{b}} P<0.05$ versus $[\mathrm{STZ}(45)]$ treated group, $\quad{ }^{\mathrm{c}} P<0.05$ versus $[\mathrm{STZ} \quad(45)+\mathrm{NaHS} \quad(10)]$ treated group, ${ }^{\mathrm{d}} P<0.05$ versus $[\mathrm{STZ} \quad(45)+\mathrm{NaHS}$ (30)] treated group, ${ }^{\mathrm{e}} P<0.05$ versus [STZ (45) + LOS (5)] treated group. $\mathrm{STZ}=$ Streptozotocin, $\mathrm{NaHS}=$ Sodium hydrosulphide, LOS $=$ Losartan, DL-p = DL-propargylglycine.

Fig. 5 Effect of NaHS and losartan on Nitrite in STZ treated rats. ${ }^{a} P<0.05$ versus vehicle treated, ${ }^{\mathrm{b}} P<0.05$ versus [STZ (45)] treated group, ${ }^{\mathrm{c}} P<0.05$ versus [STZ (45) + NaHS (10)] treated group, ${ }^{\mathrm{d} P}<0.05$ versus [STZ (45) + NaHS (30)] treated group, ${ }^{\mathrm{e}} P<0.05$ versus [STZ (45) + LOS (5)] treated group. STZ $=$ Streptozotocin, $\mathrm{NaHS}=$ Sodium hydrosulphide, LOS = Losartan, DL-p = DL-propargylglycine.

Fig. 6 Effect of NaHS and losartan on MABP in STZ treated rats. ${ }^{\mathrm{a}} P<0.05$ versus vehicle treated, ${ }^{\mathrm{b}} P<0.05$ versus [STZ (45)] treated group, ${ }^{\mathrm{c}} P<0.05$ versus [STZ (45) + NaHS (10)] treated group, ${ }^{\mathrm{d} P}<0.05$ versus $[\mathrm{STZ}$ (45) + NaHS (30)] treated group, ${ }^{\mathrm{e}} P<0.05$ versus [STZ (45) + LOS (5)] treated group. STZ = Streptozotocin, NaHS $=$ Sodium hydrosulphide, LOS = Losartan, DL-p = DL-propargylglycine.

* Correspondence: punnubansal79@gmail.com

Pharmacology Division, Department of Pharmacology, ISF College of Pharmacy, Moga 14200, Punjab, India 
The missing figure can be found below, with the correct figure legend:
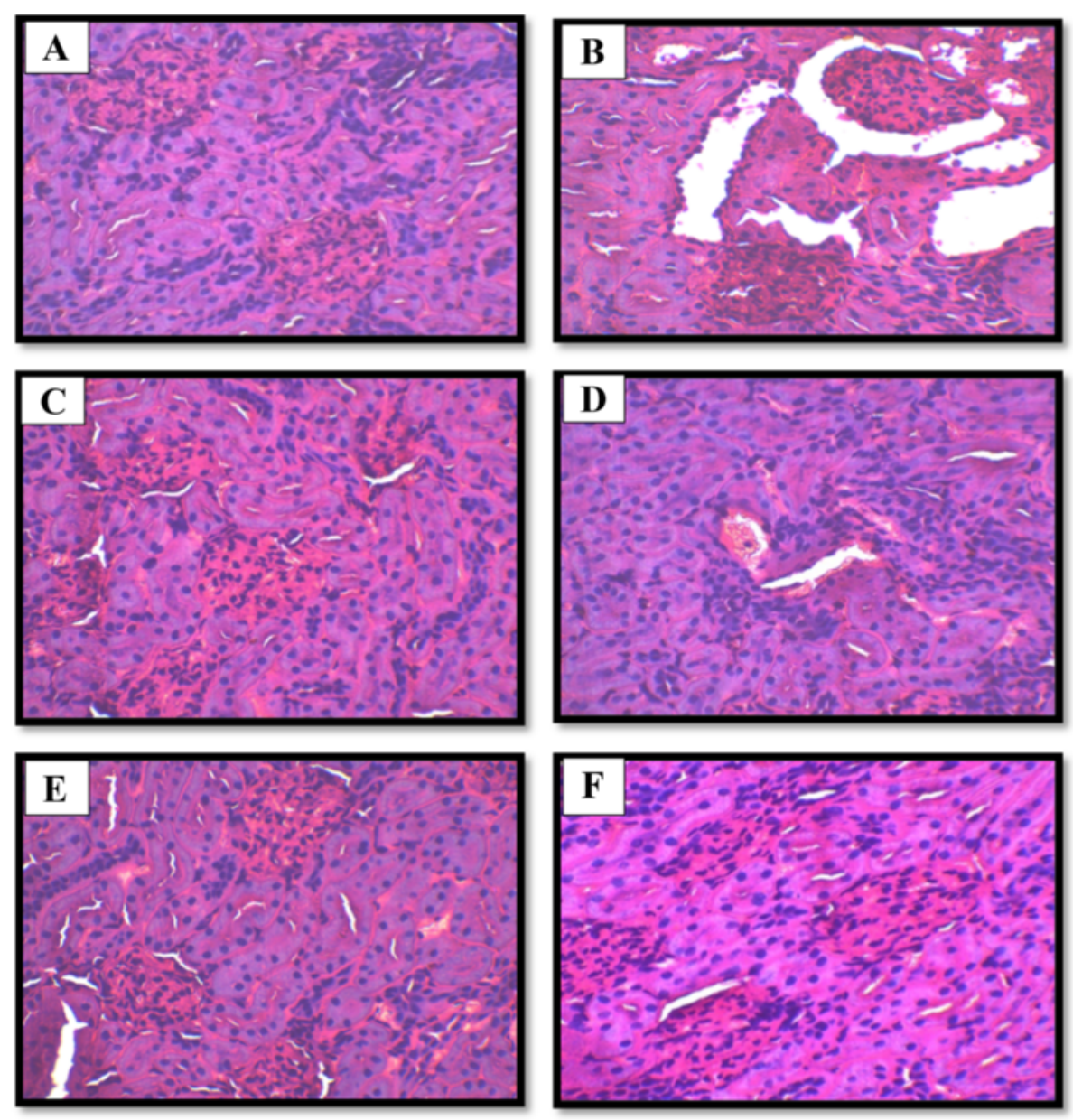

Fig. 1 Hemotoxylin-Eosin stained longitudinal section of kidneys (10x). a Normal control, b STZ treated group (45), c NaHS (30), d DL-p (10)+ $\mathrm{NaHS}$ (30), e LOS, f NaHS (10) + Losartan (5)

Received: 7 October 2015 Accepted: 8 October 2015

Published online: 22 October 2015

\section{Reference}

1. Kaur M, Sachdeva S, Bedi O, Kaur T, Kumar P. Combined effect of hydroden sulphide donor and losartan in experimental diabetic nephropathy in rats. J Diabetes Metab Disord. 2015;14:63. doi:10.1186/s40200-015-0185-7.

\section{Submit your next manuscript to BioMed Central and take full advantage of:}

- Convenient online submission

- Thorough peer review

- No space constraints or color figure charges

- Immediate publication on acceptance

- Inclusion in PubMed, CAS, Scopus and Google Scholar

- Research which is freely available for redistribution 